\title{
On the physical origin of the second solar spectrum of the Sc II line at $4247 \AA$
}

\author{
L. Belluzzi \\ Dipartimento di Astronomia e Scienza dello Spazio, University of Firenze, Largo E. Fermi 2, 50125 Firenze, Italy \\ e-mail: belluzzi@arcetri.astro.it
}

Received 21 July 2009 / Accepted 6 August 2009

\begin{abstract}
Context. The peculiar three-peak structure of the linear polarization profile shown in the second solar spectrum by the Ba II line at $4554 \AA$ has been interpreted as the result of the different contributions coming from the barium isotopes with and without hyperfine structure. In the same spectrum, a triple peak polarization signal is also observed in the Sc II line at $4247 \AA$ A. Scandium has a single stable isotope $\left({ }^{45} \mathrm{Sc}\right)$, which shows hyperfine structure due to a nuclear spin $I=7 / 2$.

Aims. We investigate the possibility of interpreting the linear polarization profile shown in the second solar spectrum by this Sc II line in terms of hyperfine structure.

Methods. A two-level model atom with hyperfine structure is assumed. Adopting an optically thin slab model, the role of atomic polarization and of hyperfine structure is investigated, avoiding the complications caused by radiative transfer effects. The slab is assumed to be illuminated from below by the photospheric continuum, and the polarization of the radiation scattered at $90^{\circ}$ is investigated.

Results. The three-peak structure of the scattering polarization profile observed in this Sc II line cannot be fully explained in terms of hyperfine structure.

Conclusions. Given the similarities between the Sc II line at $4247 \AA$ and the Ba II line at $4554 \AA$, it is not clear why, within the same modeling assumptions, only the three-peak $Q / I$ profile of the barium line can be fully interpreted in terms of hyperfine structure. The failure to interpret this Sc II polarization signal raises important questions, whose resolution might lead to significant improvements in our understanding of the second solar spectrum. In particular, if the three-peak structure of the Sc II signal is actually produced by a physical mechanism neglected within the approach considered here, it will be extremely interesting not only to identify this mechanism, but also to understand why it seems to be less important in the case of the barium line.
\end{abstract}

Key words. atomic processes - polarization - scattering - Sun: atmosphere

\section{Introduction}

The theoretical interpretation of the "second solar spectrum", namely the linearly polarized spectrum of the solar radiation coming from quiet regions close to the limb, is presently one of the most intriguing challenges in the field of solar physics. Although the basic physical process at the origin of this spectrum is clear (scattering line polarization), and although several of its properties and peculiarities have been interpreted through the theoretical approaches that have been proposed so far, our understanding of this spectrum remains rather fragmentary, and several features still elude any attempt of interpretation. The main difficulty in the interpretation of the second solar spectrum is that many physical mechanisms are capable of generating or modifying the polarization of the solar radiation, and it is an extremely complicated task to properly quantify their effects in such a complex environment as the solar atmosphere. On the other hand, our knowledge of some of these mechanisms is still rather poor, since they have received attention only recently, both from a theoretical and experimental point of view (e.g., evaluation of the depolarizing collisional rates, development of theoretical frameworks able to account for partial redistribution effects in a self-consistent way, etc.). Nevertheless, the efforts that have been made in this sense are fully justified, because a complete and correct understanding and modeling of the physics underlying the formation of the second solar spectrum will allow us to fully exploit its enormous diagnostic potential, mainly for the investigation of the magnetic fields present in the solar atmosphere (see Trujillo Bueno 2009, for a recent review).

Observations performed with instruments having sensitivities on the order of $10^{-3}-10^{-4}$ have shown in great detail the spectral richness and complexity of the second solar spectrum (see Stenflo \& Keller 1996, 1997). Among the profiles observed, those with a three-peak structure have particularly excited the interest and curiosity of the scientific community. Remarkable examples are the three-peak $Q / I$ profiles of the Ca I line at $4227 \AA$, of the Na I $\mathrm{D}_{2}$ line at $5889 \AA$, and of the $\mathrm{Ba}$ II $\mathrm{D}_{2}$ line at $4554 \AA$. The intensity spectrum and the second solar spectrum of the $\mathrm{Na}$ I and $\mathrm{Ba}$ II $\mathrm{D}_{2}$ lines are shown in the first two panels of Fig. 1.

The three-peak structure shown by the $\mathrm{Ba}$ II $\mathrm{D}_{2}$ line has been explained in terms of the presence of barium isotopes both with and without hyperfine structure (HFS) (see Stenflo 1997; Belluzzi et al. 2007). In particular, it has been shown that the two secondary peaks in the wings of the $Q / I$ profile are due to the isotopes with HFS ( $\approx 18 \%$ in abundance), while the central, higher peak is produced by the isotopes without HFS $(\approx 82 \%$ in abundance). Taking into account the effect of the HFS shown by this rather small fraction of barium isotopes, the observed threepeak profile could be reproduced to very high accuracy, even within the simplifying modeling assumption of the so-called optically thin slab model (i.e., neglecting radiative transfer effects). 

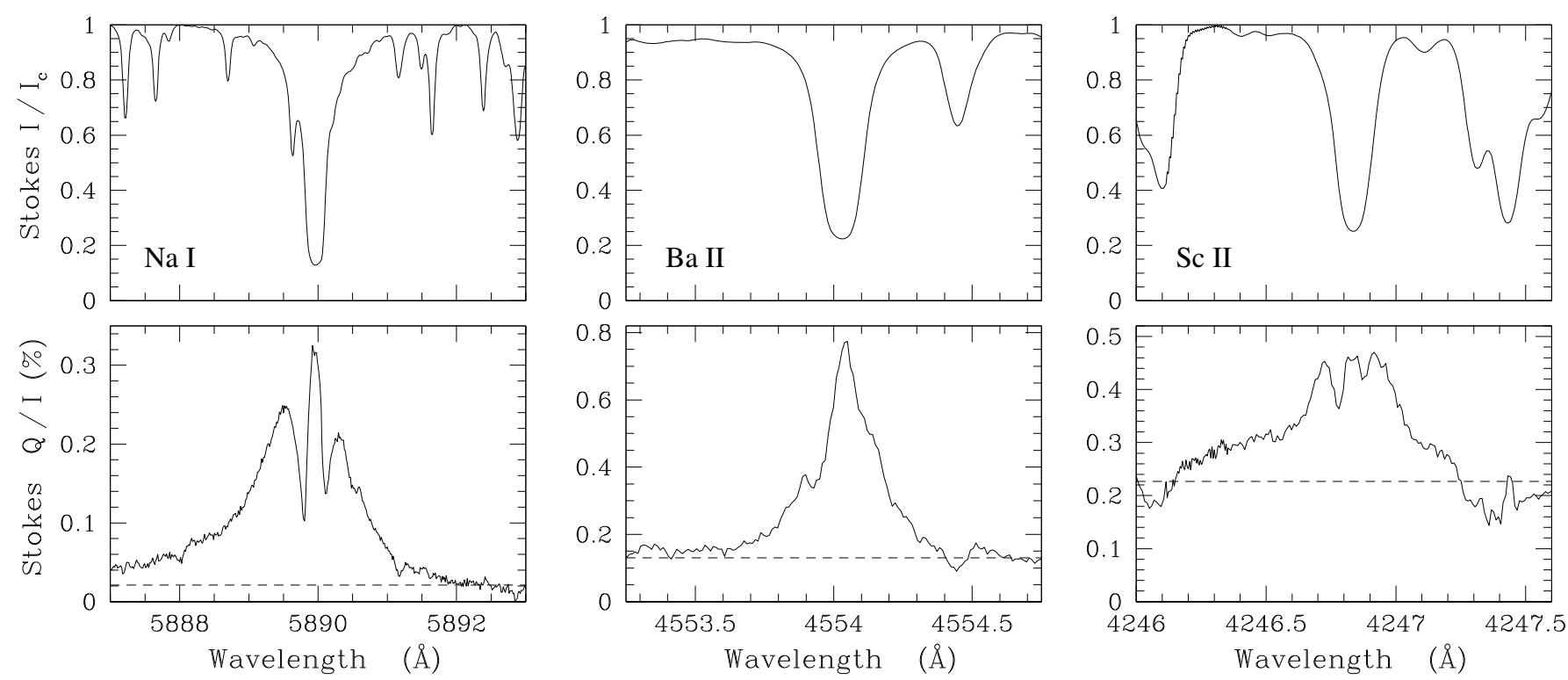

Fig. 1. Three spectral lines showing a three-peak $Q / I$ profile in the second solar spectrum: the $\mathrm{Na}$ I $\mathrm{D}_{2}$ line at $5889 \AA$ (left), the Ba II line at $4554 \AA$ (middle), and the Sc II line at $4247 \AA$ (right). The dashed line in the lower panels represents the polarization level of the continuum. All the observations are taken from Gandorfer $(2000,2002)$.

As can be observed in the right panel of Fig. 1, also the Sc II line at $4247 \AA$ shows in the second solar spectrum a three-peak $Q / I$ profile. In contrast to the $Q / I$ profile produced by the Ba II $\mathrm{D}_{2}$ line, which shows a central peak (due to the isotopes without HFS) of amplitude much larger than that of the lateral peaks, the three peaks exhibited by this scandium signal have approximately the same amplitude. This peculiarity strongly suggests that the three-peak structure of this signal might also be due to HFS, since scandium has a single stable isotope, which shows HFS (see Sect. 2.2). This possibility is strengthened by the wavelength separation between the lateral peaks being very similar to that observed in the $Q / I$ profile of the $\mathrm{Ba}_{\text {II }} \mathrm{D}_{2}$ line, and by this scandium line in the intensity spectrum being very similar to the $\mathrm{Ba}$ II $\mathrm{D}_{2}$ line. We note that these latter circumstances do not hold in the case of the $\mathrm{Na}$ I $\mathrm{D}_{2}$ line: the sodium line is much stronger and broader in the intensity spectrum, and the wavelength separation between the lateral peaks shown by the $Q / I$ profile is much larger than for either barium or scandium. Indeed, it has already been observed that the three-peak structure of the $\mathrm{Na}$ I $\mathrm{D}_{2}$ line cannot be explained only in terms of the HFS exhibited by the single stable isotope of sodium, but that its interpretation seems to require the inclusion of other physical "ingredients" such as "super-interferences" and lower level polarization (see Landi Degl'Innocenti 1998), and/or the effects of partial redistribution in frequency (see Holzreuter et al. 2005), and/or the enhancement of the line-center scattering polarization peak by vertical magnetic fields (see Trujillo Bueno et al. 2002). For the reasons explained above, we considered it worthwhile to investigate whether the peculiar three-peak structure of this scandium $Q / I$ signal might be explained in terms of HFS, within a modeling approach similar to that proposed by Belluzzi et al. (2007) for the Ba II $\mathrm{D}_{2}$ line.

\section{Formulation of the problem}

\subsection{The two-level atom with hyperfine structure}

It is well-known that hyperfine structure is produced by the influence of the nucleus on the energy levels of the atom. On the one hand, this influence is related to the nuclei of the various isotopes having slightly different volumes and masses (isotopic effect), and on the other hand, to the coupling of the nuclear spin $\boldsymbol{I}$ with the total angular momentum $\boldsymbol{J}$ of the electronic cloud (nuclear spin effect). We note that it is customary to speak about hyperfine structure tout court when referring to the nuclear spin effect only.

In the absence of magnetic fields, using Dirac's notation, the energy eigenvectors of an atomic system with HFS can be written in the form $\mid \alpha J I F f>$, where $\alpha$ represents a set of inner quantum numbers (specifying the electronic configuration and, if the atomic system is described by the $L-S$ coupling scheme, the total electronic orbital, and spin angular momenta), $F$ is the quantum number associated with the total angular momentum operator (electronic plus nuclear: $\boldsymbol{F}=\boldsymbol{J}+\boldsymbol{I}$ ), and $f$ is the quantum number associated with the projection of $\boldsymbol{F}$ along the quantization axis. It is possible to demonstrate that the HFS Hamiltonian, describing the interaction between the nuclear spin and the electronic angular momentum, can be expressed as a series of electric and magnetic multipoles (see, for example, Kopfermann 1958). In this investigation, we retain only the first two terms (magnetic-dipole and electric-quadrupole), which are given by

$$
\begin{aligned}
&\left\langle\alpha J I F f\left|H_{\mathrm{hfs}}^{(1)}\right| \alpha J I F^{\prime} f^{\prime}\right\rangle=\delta_{F F^{\prime}} \delta_{f f^{\prime}} \frac{\mathcal{A}(\alpha, J, I)}{2} K, \\
&\left\langle\alpha J I F f\left|H_{\mathrm{hfs}}^{(2)}\right| \alpha J I F^{\prime} f^{\prime}\right\rangle=\delta_{F F^{\prime}} \delta_{f f^{\prime}} \mathcal{B}(\alpha, J, I) \\
& \times \frac{3}{8}\left\{\frac{K(K+1)-\frac{4}{3} J(J+1) I(I+1)}{I(2 I-1) J(2 J-1)}\right\},
\end{aligned}
$$

where $\mathcal{A}(\alpha, J, I)$ and $\mathcal{B}(\alpha, J, I)$ are the magnetic-dipole and the electric-quadrupole HFS constants, respectively, and where

$$
K=F(F+1)-J(J+1)-I(I+1) .
$$

We describe the atomic system by means of the density matrix formalism, a robust theoretical framework very suitable for handling atomic polarization phenomena (population imbalances 
and quantum interferences between pairs of magnetic sublevels) that can be induced in the atomic system (for example by an anisotropic incident radiation field). Since the upper and lower levels of the Sc II line at $4247 \AA$ pertain to singlet terms (this is the only line of the multiplet), we consider a simple twolevel model atom with HFS. Following Landi Degl'Innocenti \& Landolfi (2004, hereafter LL04), we describe the atom through the density matrix elements

$\left\langle\alpha J I F f|\hat{\rho}| \alpha J I F^{\prime} f^{\prime}\right\rangle=\rho_{\alpha J I}\left(F f, F^{\prime} f^{\prime}\right)$,

where $\hat{\rho}$ is the density-matrix operator. We recall that the diagonal elements represent the populations of the various magnetic sublevels, while the off-diagonal elements represent the quantum interferences, or coherences, between pairs of magnetic sublevels. In the following, we work in terms of the so-called spherical statistical tensors. The conversion of the density matrix elements of Eq. (3) into the spherical statistical tensor representation is given by the relation

$$
\begin{aligned}
& { }^{\alpha J I} \rho_{Q}^{K}\left(F, F^{\prime}\right)=\sum_{f f^{\prime}}(-1)^{F-f} \sqrt{2 K+1}
\end{aligned}
$$

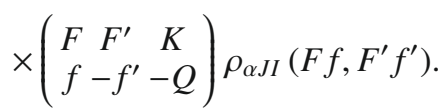

The statistical equilibrium equations (SEE), and the radiative transfer coefficients for a two-level atom with HFS, in the spherical statistical tensor representation can be found in LLO4. Here we write only the expression of the emission coefficient (in the absence of magnetic fields)

$$
\begin{aligned}
& \varepsilon_{j}(v, \mathbf{\Omega})=\frac{h v}{4 \pi} \mathcal{N}\left(2 J_{u}+1\right) A\left(\alpha_{u} J_{u} \rightarrow \alpha_{\ell} J_{\ell}\right) \\
& \times \sum_{K Q} \sum_{F_{u} F_{u}^{\prime} F_{\ell}}(-1)^{1+F_{\ell}+F_{u}^{\prime}}\left(2 F_{\ell}+1\right) \sqrt{3\left(2 F_{u}+1\right)\left(2 F_{u}^{\prime}+1\right)} \\
& \times\left\{\begin{array}{c}
J_{u} J_{\ell} 1 \\
F_{\ell} F_{u} I
\end{array}\right\}\left\{\begin{array}{c}
J_{u} J_{\ell} 1 \\
F_{\ell} F_{u}^{\prime} I
\end{array}\right\}\left\{\begin{array}{ccc}
1 & 1 & K \\
F_{u} & F_{u}^{\prime} F_{\ell}
\end{array}\right\} \\
& \times \mathcal{T}_{Q}^{K}(j, \boldsymbol{\Omega})^{\alpha_{u} J_{u} I_{\rho_{Q}}^{K}}\left(F_{u}^{\prime}, F_{u}\right) \\
& \times \frac{1}{2}\left[\Phi\left(v_{\alpha_{u} J_{u} I F_{u}, \alpha_{\ell} J_{\ell} I F_{\ell}}-v\right)+\Phi\left(v_{\alpha_{u} J_{u} I F_{u}^{\prime}, \alpha_{\ell} J_{\ell} I F_{\ell}}-v\right)^{*}\right],
\end{aligned}
$$

with $j=0,1,2,3$ (corresponding respectively to the Stokes parameters $I, Q, U$, and $V), \mathcal{N}$ the number density of atoms, $A\left(\alpha_{u} J_{u} \rightarrow \alpha_{\ell} J_{\ell}\right)$ the Einstein coefficient for spontaneous emission, $\mathcal{T}_{Q}^{K}(j, \boldsymbol{\Omega})$ a geometrical tensor (see Sect. 5.11 of LL04), and $\Phi$ the (complex) profile of the line. The indices $\ell$ and $u$ have the usual meaning of lower and upper (level), respectively.

It is important to recall that Eq. (5), and the SEE needed to find the spherical statistical tensors $\rho_{Q}^{K}\left(F_{u}, F_{u}^{\prime}\right)$ are valid under the flat-spectrum approximation. For a two-level atom with HFS, this approximation requires that the incident radiation field should be flat (i.e., independent of frequency) across a spectral interval $\Delta v$ larger than the frequency intervals among the HFS levels, and larger than the inverse lifetimes of the same levels. Given the small frequency separation between the various HFS levels (see Fig. 2), this appears to be a good approximation for the Sc II line under investigation.

\subsection{The atomic model}

Scandium has only one stable isotope $\left({ }^{45} \mathrm{Sc}\right)$, of nuclear spin $I=7 / 2$. The Sc II line at $4247 \AA$ originates from the transition between the levels $3 \mathrm{~d} 4 \mathrm{~s}{ }^{1} \mathrm{D}_{2}$ (lower level) and $3 \mathrm{~d} 4 \mathrm{p}{ }^{1} \mathrm{D}_{2}$
Table 1. Magnetic dipole and electric quadrupole HFS constants of the levels considered in this investigation.

\begin{tabular}{ccccc}
\hline \hline Isotope & $3 \mathrm{~d} 4 \mathrm{~s}$ & ${ }^{1} \mathrm{D}_{2}$ & $3 \mathrm{~d} 4 \mathrm{p}$ & ${ }^{1} \mathrm{D}_{2}$ \\
& $\mathcal{A}(\mathrm{MHz})$ & $\mathcal{B}(\mathrm{MHz})$ & $\mathcal{A}(\mathrm{MHz})$ & $\mathcal{B}(\mathrm{MHz})$ \\
\hline${ }^{45} \mathrm{Sc}$ & 128.2 & -39 & 215.7 & 18 \\
\hline
\end{tabular}

Data are taken from Villemoes et al. (1992).
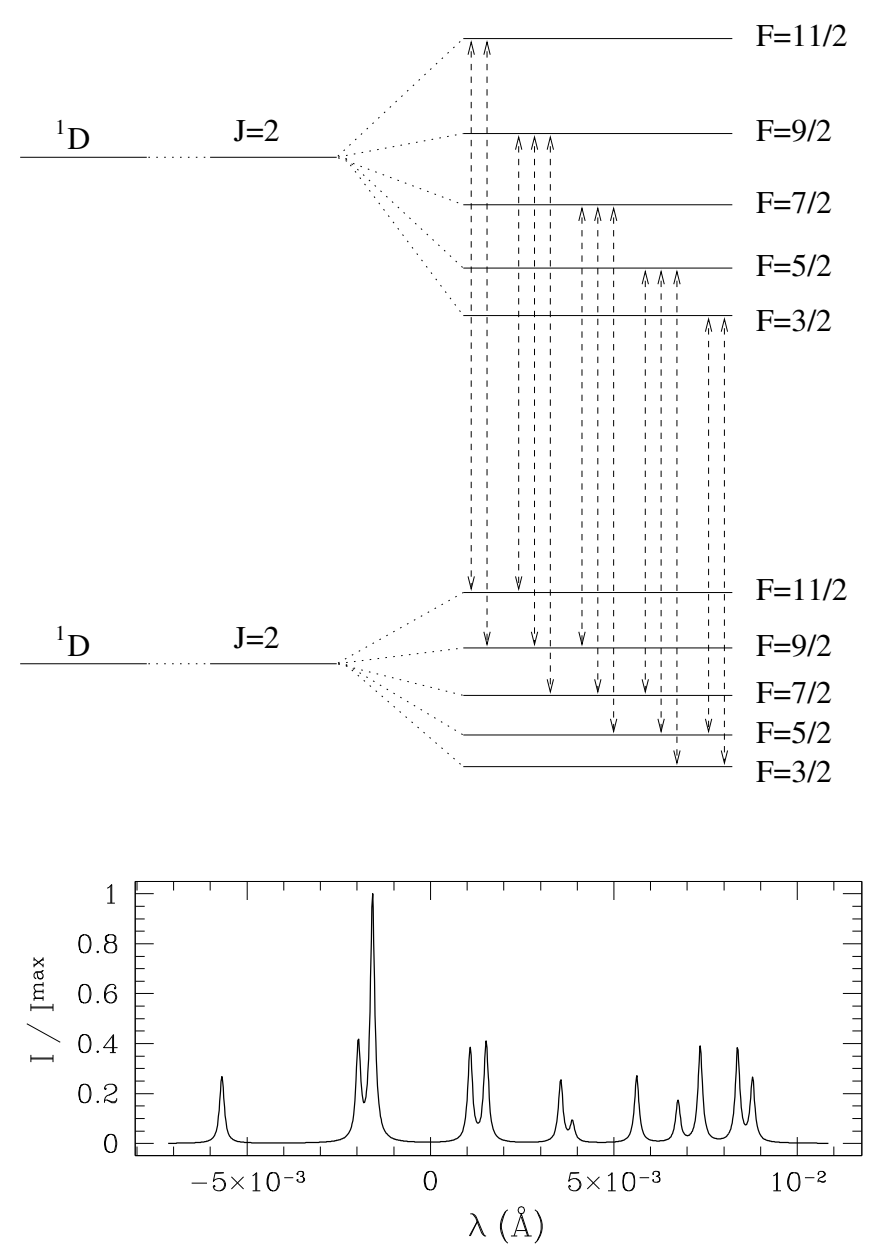

Fig. 2. Upper panel: Grotrian diagram showing the terms, the fine structure, and the hyperfine structure levels considered in the atomic model (the separation between the two $J$-levels is not on scale). The 13 HFS components of the line under investigation are drawn in the diagram. Lower panel: laboratory positions and relative strengths of the various HFS components, broadened by their natural width. Note that only twelve components are visible since two are blended. The zero of the wavelength scale is at $4246.82 \AA$.

(upper level). The Einstein coefficient for spontaneous emission is $A=1.29 \times 10^{8} \mathrm{~s}^{-1}$ (Ralchenko et al. 2008). Because of HFS, each $J$-level splits into $5 F$-levels, and the spectral line under investigation consists of 13 HFS components (see the upper panel of Fig. 2). We use the energies of the $J$-levels provided by Ralchenko et al. (2008), while we calculate the energies of the various HFS levels by applying Eq. (1), using the values of the magnetic dipole and of the electric quadrupole HFS constants listed in Table 1. The laboratory positions and the relative strengths of the various HFS components are shown in the lower panel of Fig. 2. 


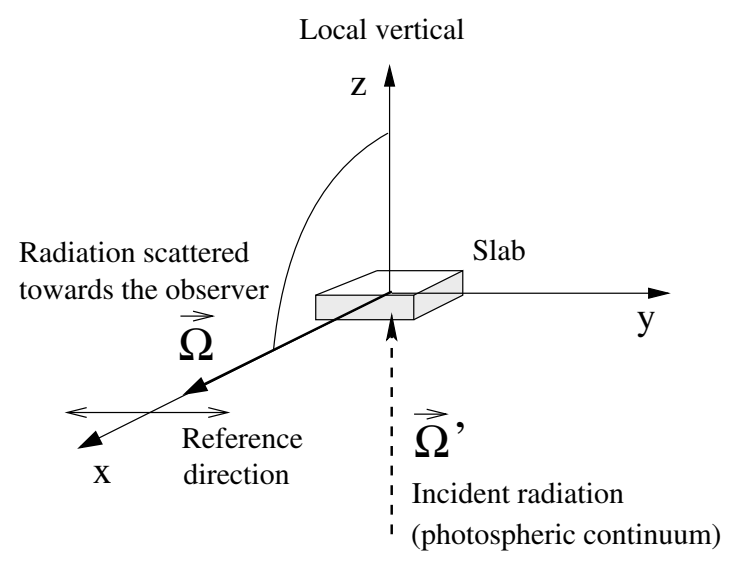

Fig. 3. Geometry of the problem being investigated.

Despite the simplicity of the atomic model considered (twolevel atom), because of the high values of the quantum numbers involved, the SEE form a set of 3200 equations in 3200 unknowns (the various spherical statistical tensors ${ }^{\alpha J I} \rho_{Q}^{K}\left(F f, F^{\prime} f^{\prime}\right)$ of the upper and lower levels). To reduce the amount of numerical calculations involved in the solution of this set of equations, we limit ourselves to considering only the spherical statistical tensors with $K \leq 2$, thus reducing the SEE to a set of 278 equations. Because of the low value of the anisotropy factor that we assume for the radiation field (see following subsection), the statistical tensors of higher rank are found to be almost two orders of magnitude smaller, so that the results obtained within this approximation do not show any appreciable difference with respect to those obtained by carrying out the complete calculations.

\subsection{The optically thin slab model}

To emphasize the atomic aspects involved in the problem, avoiding complications due to radiative transfer effects, we consider an optically thin slab of Sc II ions located $1000 \mathrm{~km}$ above the solar surface (as defined in Sect. 12.3 of LL04), and we assume that the slab is illuminated from below by the photospheric continuum (see Fig. 3). Based on these hypotheses, the atomic polarization can be calculated by solving the SEE directly for the given incident continuum radiation field. We remark that this model is basically the same as that employed by Belluzzi et al. (2007) to reproduce the $Q / I$ profile of the $\mathrm{Ba} \mathrm{II}_{2}$ line. We assume that the continuum is unpolarized and cylindrically symmetric about the local vertical. Under these assumptions, taking a reference system with the $z$-axis (the quantization axis) directed along the vertical, it can be shown that only two components of the radiation field tensor, in terms of which we describe the incident continuum, are non-vanishing

$$
\begin{aligned}
& J_{0}^{0}(v)=\oint \frac{\mathrm{d} \Omega}{4 \pi} I(v, \mu), \quad \text { and } \\
& J_{0}^{2}(v)=\frac{1}{2 \sqrt{2}} \oint \frac{\mathrm{d} \Omega}{4 \pi}\left(3 \mu^{2}-1\right) I(v, \mu),
\end{aligned}
$$

where $\mu$ is the cosine of the heliocentric angle. The former quantity is the mean intensity of the radiation field (averaged over all directions), the second quantifies the anisotropy of the radiation field (imbalance between vertical and horizontal illumination). The mean intensity of the radiation field can also be expressed in terms of the average number of photons per mode, $\bar{n}$, while the anisotropy degree is often quantified through the so-called

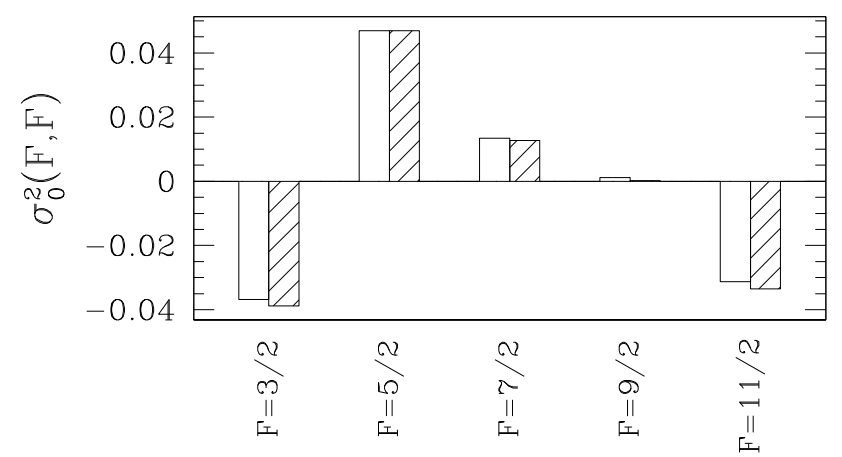

Fig. 4. Fractional atomic alignment $\sigma_{0}^{2}(F, F)=\rho_{0}^{2}(F, F) / \rho_{0}^{0}(F, F)$ in the lower (white columns) and upper (shaded columns) levels. Note that as far as the fractional atomic alignment of the lower level is concerned, the quantity $-\sigma_{0}^{2}\left(F_{\ell}, F_{\ell}\right)$ is plotted. The values have been obtained by solving the SEE for the values of $\bar{n}$ and $w$ quoted in the text, and in the absence of collisions, magnetic fields, and stimulation effects.

anisotropy factor, $w$. These new, non-dimensional quantities are related to $J_{0}^{0}$ and $J_{0}^{2}$ by the equations

$\bar{n}(v)=\frac{c^{2}}{2 h v^{3}} J_{0}^{0}(v), \quad w(v)=\sqrt{2} \frac{J_{0}^{2}(v)}{J_{0}^{0}(v)}$.

We calculate the values of $\bar{n}(v)$ and $w(v)$ of the photospheric continuum following Sect. 12.3 of LL04 taking $h=1000 \mathrm{~km}$, and using the values of the disk center intensities, and of the limbdarkening coefficients given by Pierce (2000). At the height of the slab, and at the frequency of the spectral line under investigation $\left(4247 \AA\right.$ ) we find $\bar{n}=0.158 \times 10^{-2}$, and $w=0.189$.

Taking into account the atomic weight of scandium, assuming a temperature of $6000 \mathrm{~K}$, and neglecting microturbulent velocity, we obtain for this line a Doppler width, $\Delta \lambda_{\mathrm{D}}$, given by

$\Delta \lambda_{\mathrm{D}}=\frac{\lambda_{0}}{c} w_{\mathrm{T}}=\frac{\lambda_{0}}{c} \sqrt{\frac{2 k_{\mathrm{B}} T}{M}}=21 \mathrm{~m} \AA$,

where $w_{\mathrm{T}}$ is the thermal velocity, $T$ the kinetic temperature, $M$ the mass of the ion, and $k_{\mathrm{B}}$ the Boltzmann constant.

\subsection{Polarization of the emergent spectral line radiation}

Once the SEE have been written down and solved numerically, we can calculate the radiative transfer coefficients according to the equations of Sect. 7.9 of LL04. We consider the radiation scattered by the slab at $90^{\circ}$, and we take the reference direction for positive $Q$ parallel to the slab (see Fig. 3). In the case of a tangential observation, under the approximation of a weakly polarizing atmosphere $\left(\varepsilon_{I} \gg \varepsilon_{Q}, \varepsilon_{U}, \varepsilon_{V} ; \eta_{I} \gg \eta_{Q}, \eta_{U}, \eta_{V}, \rho_{Q}, \rho_{U}, \rho_{V}\right)$, it can be shown that the emergent fractional polarization is given by (see Trujillo Bueno 2003)

$\frac{X(v, \boldsymbol{\Omega})}{I(v, \boldsymbol{\Omega})} \approx \frac{\varepsilon_{X}(v, \boldsymbol{\Omega})}{\varepsilon_{I}(v, \boldsymbol{\Omega})}-\frac{\eta_{X}(v, \boldsymbol{\Omega})}{\eta_{I}(v, \boldsymbol{\Omega})} \quad$ with $X=Q, U, V$.

The first and second terms in the right-hand side of Eq. (9) represent the contribution to the emergent radiation due to processes of selective emission and selective absorption (dichroism) of polarization components, respectively.

We note that while the lower level of $\mathrm{Ba}$ II $\mathrm{D}_{2}$, with $J=1 / 2$, can carry alignment only because of the presence of HFS, the lower level of this scandium line, with $J=2$, can be polarized 

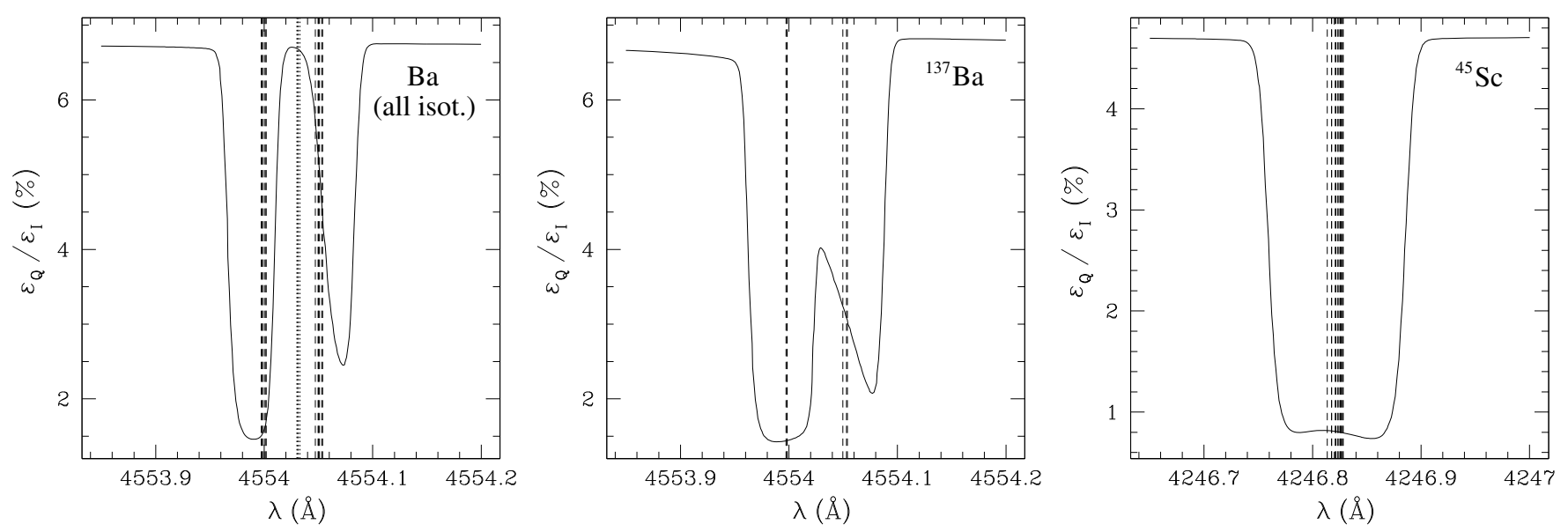

Fig. 5. Left panel: fractional polarization profile for the Ba II line at $4554 \AA$, obtained by applying Eq. (10) (without continuum). The values of $\bar{n}$, $w$, and $\Delta \lambda_{\mathrm{D}}$ are calculated for a slab of barium ions at $6000 \mathrm{~K}$, located at a height of $1000 \mathrm{~km}\left(\bar{n}=0.225 \times 10^{-2}, w=0.176, \Delta \lambda_{\mathrm{D}}=13 \mathrm{~m} \AA\right)$. The dashed lines show the wavelength position of the HFS components of ${ }^{135} \mathrm{Ba}$ and ${ }^{137} \mathrm{Ba}$, and the dotted lines the wavelength position of the fine structure components of the isotopes without HFS. Middle panel: same as left panel but assuming that only the isotope ${ }^{137} \mathrm{Ba}$ (with HFS) is present (100\% in abundance). The dashed lines show the wavelength position of the various HFS components. Right panel: fractional polarization profile for the Sc II line at $4247 \AA$, obtained by applying Eq. (10) and assuming for $\bar{n}, w$, and $\Delta \lambda_{\mathrm{D}}$ the values quoted in Sect. 2.3 (corresponding to a slab at $6000 \mathrm{~K}$, located $1000 \mathrm{~km}$ above the solar surface). The dashed lines show the wavelength position of the various HFS components.

also neglecting HFS. As a consequence, while in the case of the $\mathrm{Ba}$ II $\mathrm{D}_{2}$ line the lower level results to be significantly less polarized than the upper level (see Belluzzi et al. 2007), in this scandium line the upper and lower levels carry the same amount of atomic polarization ${ }^{1}$ (see Fig. 4). Although the contribution of dichroism is thus expected to be more important in this line than in the $\mathrm{Ba}$ II $\mathrm{D}_{2}$ line, we start our investigation by neglecting it. We therefore calculate the polarization of the radiation emerging from the slab by means of the equation

$\frac{X(v, \boldsymbol{\Omega})}{I(v, \boldsymbol{\Omega})} \approx \frac{\varepsilon_{X}(v, \boldsymbol{\Omega})}{\varepsilon_{I}(v, \boldsymbol{\Omega})}$.

Some results obtained taking into account dichroism effects (i.e., applying Eq. (9)) will be shown in Sect. 3.4.

We recall that the emission coefficient given in Eq. (5) includes only line processes. To reproduce, albeit qualitatively, the observed profile, we need to add the contribution of the continuum. Assuming that the continuum is constant across the line, we have

$\frac{X(v, \boldsymbol{\Omega})}{I(v, \boldsymbol{\Omega})} \approx \frac{\varepsilon_{X}^{\ell}(v, \boldsymbol{\Omega})+\varepsilon_{X}^{\mathrm{c}}}{\varepsilon_{I}^{\ell}(v, \boldsymbol{\Omega})+\varepsilon_{I}^{\mathrm{c}}}$,

where the superscripts " $c$ " and " $\ell$ " denote that the corresponding quantities refer to continuum and line processes, respectively. The quantities $\varepsilon_{X}^{c}$ and $\varepsilon_{I}^{c}$ are considered to be free parameters in the problem that are adjusted to reproduce the observed polarization profile.

We point out that the three-peak structure of the $Q / I$ profile shown by the $\mathrm{Ba}$ II $\mathrm{D}_{2}$ line could be reproduced by Belluzzi et al. (2007) within the constraints of the same modeling assumptions and approximations outlined above. However, we note that this ionized scandium line is a rather strong spectral line: as for $\mathrm{Ba}$ II $\mathrm{D}_{2}$, the wings of this line originate in the photosphere, while the line core originates in the high photosphere/low chromosphere. The optically thin slab model considered in this paper is therefore just a first order approximation. Nevertheless, as

${ }^{1}$ Collisions will be neglected throughout this investigation. previously stated, it allows us to take into account in a very rigorous way the relevant atomic aspects of the problem, avoiding the complications produced by radiative transfer effects.

\section{Results}

\subsection{Three-peak structure of the $\mathrm{Ba} / / \mathrm{D}_{2}$ line $\mathrm{Q} / \mathrm{I}$ profile}

As discussed in Belluzzi et al. (2007), the presence of barium isotopes both with and without HFS is understood to be important in explaining the three-peak structure of the $Q / I$ profile shown in the second solar spectrum by the $\mathrm{Ba}$ II $\mathrm{D}_{2}$ line. In particular, the central peak is due to the isotopes without HFS, while the two lateral peaks seem to be caused by the depolarizing effect of the isotopes with HFS $\left({ }^{135} \mathrm{Ba}\right.$ and $\left.{ }^{137} \mathrm{Ba}\right)$, combined with the effect of the continuum, which depolarizes the wings of the line. The fractional polarization profile plotted in the left panel of Fig. 5 clearly shows the depolarizing effect of the isotopes with HFS, as well as the polarization enhancement at line center due to the isotopes without HFS. This profile was obtained by applying Eq. (10) (i.e., neglecting the continuum) to the case of the $\mathrm{Ba}$ II $\mathrm{D}_{2}$ line, assuming for $\bar{n}, w$, and $\Delta \lambda_{\mathrm{D}}$ the values corresponding to a slab of barium ions at $6000 \mathrm{~K}$, located $1000 \mathrm{~km}$ above the solar surface. Starting from this fractional polarization profile, and adding the contribution of the continuum, Belluzzi et al. (2007) were able to obtain a good fit to the observed threepeak $Q / I$ profile.

The fundamental role of the isotopes without HFS in producing the higher central peak of the observed linear polarization profile can be clearly appreciated from the middle panel of Fig. 5. Here the same fractional polarization profile as in the left panel of Fig. 5 (without continuum) is plotted assuming that only the isotope ${ }^{137} \mathrm{Ba}$ (with HFS) is present (100\% in abundance). Comparing the two profiles, it is noticeable that although a polarization enhancement can be observed at line center also when only ${ }^{137} \mathrm{Ba}$ is present, this is much larger when the isotopes without HFS are also taken into account. In particular, if these isotopes are considered, the polarization at line center reaches the same value as in the far wings. This is an important peculiarity, 

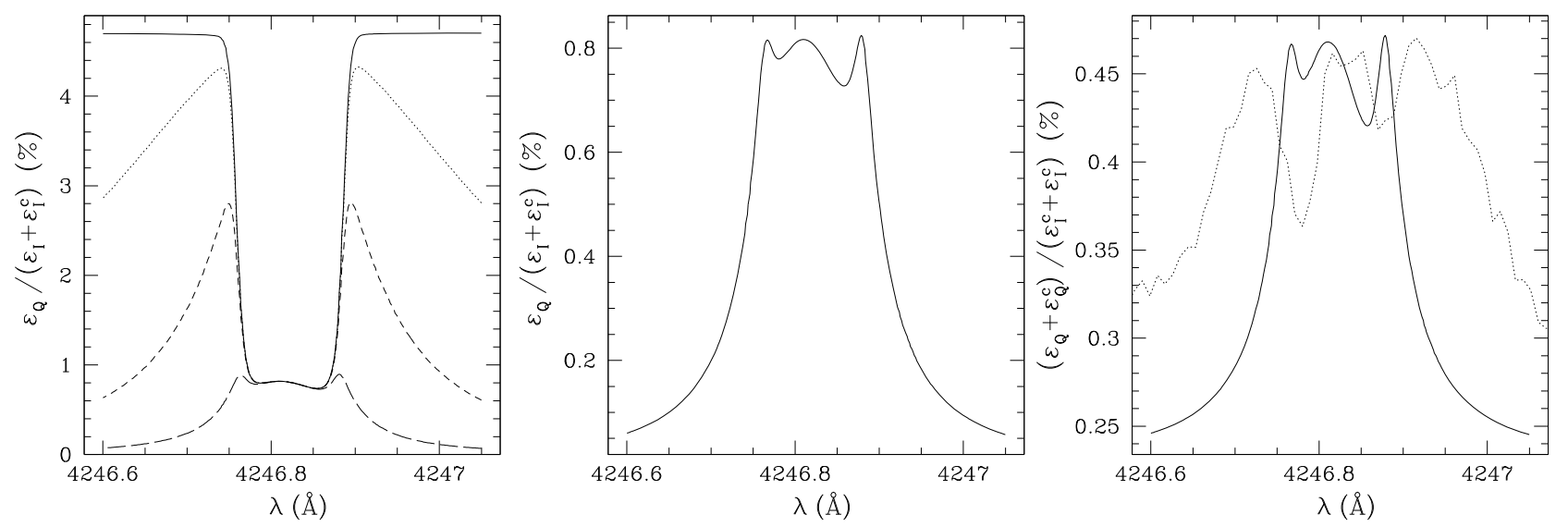

Fig. 6. Left panel: theoretical profiles obtained including the continuum contribution to the intensity. The various profiles have been obtained by assuming $\varepsilon_{I}^{\mathrm{c}} / \varepsilon_{I}^{\max }=10^{-5}$ (dotted line), $10^{-4}$ (dashed line), and $10^{-3}$ (long-dashed line). The profile plotted with solid line has been obtained without continuum, and it is identical to the one shown in the right panel of Fig. 5. The other parameters $\left(\bar{n}, w\right.$, and $\left.\Delta \lambda_{\mathrm{D}}\right)$ have the same values as in the right panel of Fig. 5. Middle panel: profile obtained by setting $\varepsilon_{I}^{\mathrm{c}} / \varepsilon_{I}^{\max }=1.2 \times 10^{-3}$. Right panel: theoretical polarization profile obtained by applying Eq. (11), setting $w=0.11, \varepsilon_{I}^{\mathrm{c}} / \varepsilon_{I}^{\max }=2.4 \times 10^{-3}, \varepsilon_{Q}^{\mathrm{c}} / \varepsilon_{I}^{\mathrm{c}}=0.23 \times 10^{-2}$, and $\Delta \lambda_{\mathrm{D}}=21 \mathrm{~m} \AA$ (solid line), and the observed profile (dotted line) taken from Gandorfer (2002).

since in this case there is no way, by adding the continuum, to obtain three peaks of the same amplitude (such as those observed in the Sc II line). On the other hand, this would be possible if, hypothetically, only the isotope ${ }^{137} \mathrm{Ba}$ were present.

\subsection{Three-peak structure of the Sc I/ $4247 \AA$ line $Q / /$ profile}

As previously pointed out, scandium has a single stable isotope, with HFS, which is consistent with the interpretation of this scandium signal, showing three peaks of the same amplitude, in terms of HFS. Applying Eq. (10), and using the values previously calculated for $\bar{n}, w$, and $\Delta \lambda_{\mathrm{D}}$, we find the fractional polarization profile shown in the right panel of Fig. 5. The profile clearly shows the depolarizing effect of $\mathrm{HFS}^{2}$, but the polarization enhancement at line-center, which produces the central peak, is by far less evident than in the case of ${ }^{137} \mathrm{Ba}$. On the one hand, this is because the HFS components of ${ }^{137} \mathrm{Ba}$ are gathered into two well separated groups (because of the large HFS splitting of the ground level), while the HFS components of scandium are gathered into a single group at line center, spreading out over a narrow wavelength interval of about $15 \mathrm{~m} \AA$ (see the middle and right panels of Fig. 5, and Fig. 2). On the other hand, the smaller Doppler width assumed for barium (much heavier than scandium) contributes to making the line-core enhancement of the corresponding fractional polarization profile far more evident.

As in the case of barium, to reproduce the observed profile, it is necessary to include the effect of the continuum. By so doing, the value of the fractional polarization decreases in the wings of the line, and a two-peak profile with a small hump at line center is obtained. The left panel of Fig. 6 shows the profiles that result when assuming different values of $\varepsilon_{I}^{\mathrm{c}} / \varepsilon_{I}^{\max }$, where $\varepsilon_{I}^{\max }$ is the maximum value of $\varepsilon_{I}^{\ell}$ in the wavelength range considered. A value of $\varepsilon_{I}^{\mathrm{c}} / \varepsilon_{I}^{\max }$ on the order of $10^{-3}$ is needed to obtain similar polarization values in the wing peaks and the line-core. Because

\footnotetext{
2 Note that if HFS had been neglected, the fractional polarization profile calculated considering only the line processes (no continuum) would have been constant with wavelength, as it is clear from Belluzzi et al. (2007), where the results obtained considering only ${ }^{138} \mathrm{Ba}$ (without HFS) are shown.
}

of the small enhancement exhibited by the fractional polarization profile at line center, by setting $\varepsilon_{I}^{\mathrm{c}} / \varepsilon_{I}^{\max }=1.2 \times 10^{-3}$ we actually obtain a profile with three peaks of the same amplitude (see the middle panel of Fig. 6).

To reproduce the amplitude of the central peak $(\approx 0.46 \%$, according to the observation of Gandorfer 2002), and the polarization level of the continuum at the wavelength of this line $(\approx 0.23 \%)$, we have to set $w=0.11$ and $\varepsilon_{Q}^{\mathrm{c}} / \varepsilon_{I}^{\mathrm{c}}=0.23 \times 10^{-2}$. Assuming that $\varepsilon_{I}^{\mathrm{c}} / \varepsilon_{I}^{\max }=2.4 \times 10^{-3}$ (which is a rather realistic value for a spectral line such as that under investigation), we obtain the theoretical profile plotted in the right panel of Fig. 6. This profile indeed shows a three-peak structure, but its width can be immediately noticed to be much smaller than that of the observed profile. In particular, in the observed profile the wavelength separations between the two dips and between the two lateral peaks are about $90 \mathrm{~m} \AA$ and $190 \mathrm{~m} \AA$, respectively, while in the theoretical profile they are about $75 \mathrm{~m} \AA$ and $110 \mathrm{~m} \AA$, respectively. Moreover, while the amplitude of the dip at longer wavelengths (the "red" dip) is very similar to the observed one, the amplitude of the "blue" dip is much smaller.

The only way to increase the width of the theoretical profile is to assume a larger value of the Doppler width. In the case of barium, to reproduce the observed profile it was necessary to assume a Doppler width of $30 \mathrm{~m} \AA$, corresponding to a temperature of $6000 \mathrm{~K}$ and a microturbulent velocity of $1.8 \mathrm{~km} \mathrm{~s}^{-1}$ (see Belluzzi et al. 2007). In the left panel of Fig. 7, the barium fractional polarization profiles, obtained by assuming $\Delta \lambda_{\mathrm{D}}=13 \mathrm{~m} \AA$ (dotted line) and $\Delta \lambda_{\mathrm{D}}=30 \mathrm{~m} \AA$ (solid line) are shown: increasing the Doppler width, the wavelength separation between the two dips is increased, but the two-dip structure of the profile is not lost. In the middle panel of Fig. 7, we plot the fractional polarization profiles of the scandium line, obtained by assuming $\Delta \lambda_{\mathrm{D}}=21 \mathrm{~m} \AA$ (dotted line) and $\Delta \lambda_{\mathrm{D}}=33 \mathrm{~m} \AA$ (solid line), the latter corresponding to a temperature of $6000 \mathrm{~K}$ and a microturbulent velocity of $1.8 \mathrm{~km} \mathrm{~s}^{-1}$. Increasing the Doppler width, the fractional polarization profile shows a significantly larger depolarizing feature, but the small polarization enhancement at line core, and therefore the three-peak structure of the polarization profile obtained by adding the continuum, are almost completely lost (see the middle and right panels of Fig. 7). 

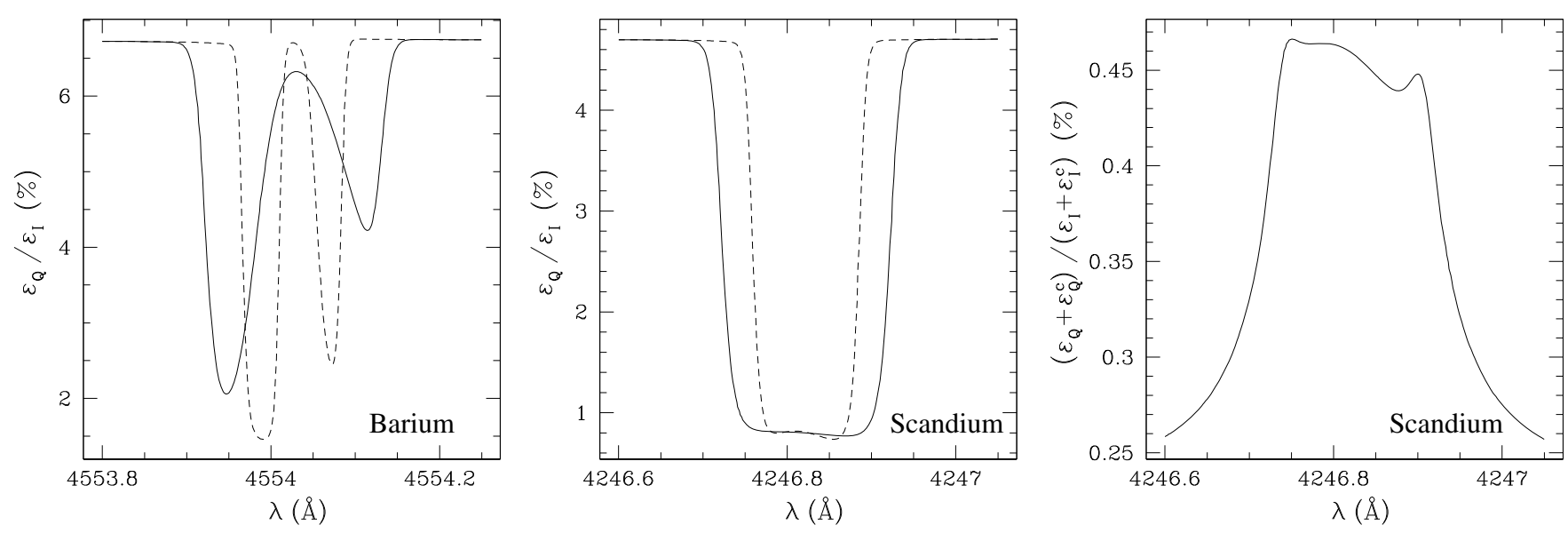

Fig. 7. Left panel: fractional polarization profiles for the Ba II line at $4554 \AA$, obtained by applying Eq. (10) (without continuum), assuming $\Delta \lambda_{\mathrm{D}}=13 \mathrm{~m} \AA$ (dotted line), and $\Delta \lambda_{\mathrm{D}}=30 \mathrm{~m} \AA$ (solid line). The other free parameters have the same values as in the left panel of Fig. 5 . The dotted-line profile is the same as in the left panel of Fig. 5. Middle panel: fractional polarization profiles for the Sc II line at $4247 \AA$, obtained by applying Eq. (10), assuming $\Delta \lambda_{\mathrm{D}}=21 \mathrm{~m} \AA$ (dotted line), and $\Delta \lambda_{\mathrm{D}}=33 \mathrm{~m} \AA$ (solid line). The other free parameters have the same values as in the right panel of Fig. 5. The dotted-line profile is the same as in the right panel of Fig. 5. Right panel: theoretical polarization profile for the Sc II line, obtained by applying Eq. (11) and setting $\Delta \lambda_{\mathrm{D}}=33 \mathrm{~m} \AA, w=0.11, \varepsilon_{I}^{\mathrm{c}} / \varepsilon_{I}^{\max }=2.1 \times 10^{-3}$, and $\varepsilon_{Q}^{\mathrm{c}} / \varepsilon_{I}^{\mathrm{c}}=0.23 \times 10^{-2}$.
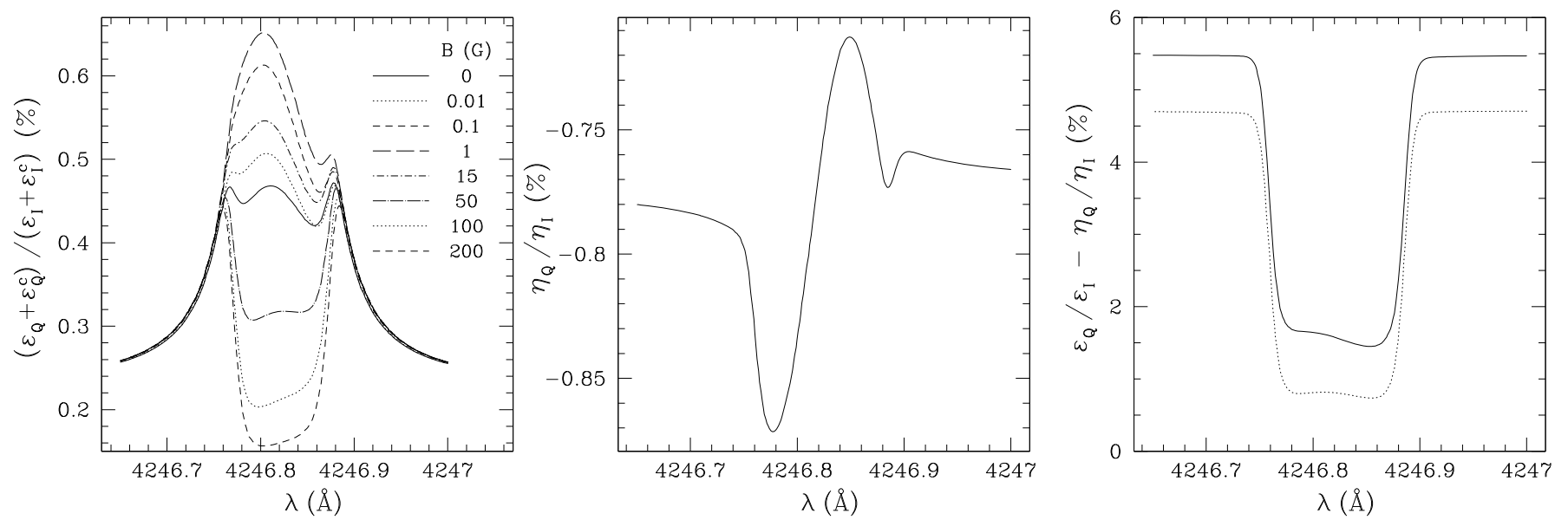

Fig. 8. Left panel: theoretical polarization profiles obtained in the presence of a unimodal microturbulent magnetic field of various intensities. All the profiles are calculated by using Eq. (11), assuming for the free parameters the same values as in the right panel of Fig. 6. The solid-line profile is the same as in the right panel of Fig. 6. Middle panel: $\eta_{Q} / \eta_{I}$ profile calculated considering only the line processes (no continuum). The free parameters have the same values as in the right panel of Fig. 5. Right panel: theoretical profiles obtained in the absence of continuum taking into account (solid line) and neglecting (dotted line) dichroism. The profiles were obtained by applying Eqs. (9) and (10), respectively. The free parameters have the same values as in the right panel of Fig. 5. The dotted-line profile is the same as in the right panel of Fig. 5.

\subsection{The effect of a unimodal microturbulent magnetic field}

The left panel of Fig. 8 shows the theoretical linear polarization profiles obtained according to Eq. (11), in the presence of a unimodal microturbulent magnetic field of various intensities. A useful estimate of the spectral line sensitivity to the Hanle effect can be obtained by calculating the critical fields of the upper and lower levels (see, for example, Trujillo Bueno 2001)

$B_{\mathrm{c}}^{\mathrm{up}}=\frac{1.137 \times A}{g_{u}} \approx 15 \mathrm{G}, \quad B_{\mathrm{c}}^{\mathrm{low}}=\frac{1.137 \times A \bar{n}}{g_{\ell}} \approx 20 \mathrm{mG}$,

where the Einstein coefficient for spontaneous emission must be expressed in units of $10^{7} \mathrm{~s}^{-1}$. In agreement with the previous estimate, we observe an increase of the polarization, due to the lower-level Hanle effect, for magnetic fields between $10 \mathrm{mG}$ and $1 \mathrm{G}$. The polarization is found to increase by a larger amount at the wavelength positions of both the central peak and the blue dip, than of the red dip. If the continuum is changed, so that the wing peaks have the same amplitude as the central peak, a three-peak structure might possibly be recovered, but a red dip much deeper than the blue one would be found, in disagreement with the observation. We point out that the lower-level Hanle effect is particularly evident in this scandium line because, as previously observed, the lower level carries the same amount of atomic polarization as the upper level (in the $\mathrm{Ba}$ II $\mathrm{D}_{2}$ line, where the lower level is much less polarized than the upper level, this effect cannot be observed).

If the magnetic field is further increased, the upper level Hanle effect becomes dominant, and a depolarization at the line core is observed. We note that for magnetic fields on the order of $100 \mathrm{G}$, the polarization at line center becomes lower than in the far wings (i.e., lower than the continuum), although always 
remaining positive. A saturation regime is reached for fields of about $200 \mathrm{G}$. Even though for fields on the order of $50 \mathrm{G}$, a threepeak structure can still be recovered by adjusting the parameters ( $w$ and $\varepsilon_{I}^{\mathrm{c}}$ ), nevertheless the observed profile cannot be reproduced. In particular, the width of the theoretical profile does not increase appreciably with the magnetic field intensity, so that the disagreement with the observation previously discussed cannot be solved.

In conclusion, within our modeling assumptions, which also include the effect of a unimodal microturbulent magnetic field, it is not possible to reproduce the observed $Q / I$ profile.

\subsection{Results obtained including dichroism}

In the middle panel of Fig. 8 , the $\eta_{Q} / \eta_{I}$ profile is plotted. In the line-core, it exhibits an anti-symmetrical shape, while in the far wings, because of the presence of lower level polarization, it reaches an asymptotic value that differs from zero. The profile obtained by applying Eq. (9) is plotted with a solid line in the right panel of Fig. 8. Comparing this profile with the one previously obtained by means of Eq. (10) (plotted with a dotted line in the same figure), an overall shift towards higher values of polarization is discernible, in addition to a slightly different substructure in the line-core, that, however, does not provide a more accurate fit to the observed profile. The inclusion of dichroism does not modify the width of the profile. By also including dichroism, if we increase the width of the profile by assuming a larger value of the Doppler width, the three-peak structure of the profile is found to be lost.

\section{Conclusions}

In terms of the same modeling approximations adopted by Belluzzi et al. (2007) to reproduce the three-peak $Q / I$ profile observed in the second solar spectrum of the $\mathrm{Ba} \mathrm{II} \mathrm{D}_{2}$ line, it is impossible to reproduce the similar three-peak linear polarization profile exhibited in the same spectrum by the Sc II line at $4247 \AA$. This is a surprising result given the similarities between the two lines in the intensity spectrum, and that the only stable isotope of scandium shows hyperfine structure, which is the physical aspect responsible for the triple peak structure of the $\mathrm{Ba}$ II $\mathrm{D}_{2}$ line $Q / I$ profile. The possibility of reproducing the polarization signal of this ionized scandium line in terms of hyperfine structure was also suggested by the three peaks having the same amplitude, a circumstance that appears to be in perfect agreement with the absence of scandium isotopes without hyperfine structure. We are indeed able to obtain a $Q / I$ profile with three peaks of the same amplitude, but unfortunately its width is found to be considerably smaller than that of the observed profile. Moreover, while the red dip can be reproduced quite well, the depth of the blue one is much smaller than in the observation. The only way to increase the width of the profile is to assume a larger Doppler width, but, in this case, the three peak structure is rapidly lost. We investigated the effect of a microturbulent magnetic field, as well as the effect of dichroism, but they both do not seem to be able to explain the disagreement between our theoretical result and the observation. Nevertheless, it is important to point out that the lower level of this line, being polarizable irrespective of the hyperfine structure $\left(J_{\ell}=2\right)$, is found to be as polarized as the upper level (depolarizing collisions have been neglected in this investigation). This circumstance explains why the lower-level Hanle effect is so clearly evident in this line. In conclusion, the physical origin of the observed threepeak $Q / I$ profile of this scandium line does not seem to be the mere presence of hyperfine structure. We may speculate that an additional physical mechanism is at work (such as, for instance, PRD effects). However, if this turns out to be the case, it would be of interest to investigate the influence of this physical mechanism on the $Q / I$ profile of the $\mathrm{Ba}$ II $\mathrm{D}_{2}$ line, whose triple peak structure could be interpreted solely in terms of hyperfine structure.

Acknowledgements. The author wishes to express his gratitude to Egidio Landi Degl'Innocenti and Javier Trujillo Bueno for several scientific discussions, and for helpful comments and suggestions.

\section{References}

Belluzzi, L., Trujillo Bueno, J., \& Landi Degl'Innocenti, E. 2007, ApJ, 666, 588 Gandorfer, A. 2000, The Second Solar Spectrum: A High Resolution Polarimetric Survey of Scattering Polarization at the Solar Limb in Graphical Representation, Vol. I: $4625 \AA$ to $6995 \AA$ (Zürich: vdf ETH)

Gandorfer, A. 2002, The Second Solar Spectrum: A High Resolution Polarimetric Survey of Scattering Polarization at the Solar Limb in Graphical Representation, Vol. II: $3910 \AA$ to $4630 \AA$ (Zürich: vdf ETH)

Holzreuter, R., Fluri, D. M., \& Stenflo, J. O. 2005, A\&A, 434, 713

Kopfermann, H. 1958, Nuclear Moments (New York: Academic Press)

Landi Degl'Innocenti, E. 1998, Nature, 392, 256

Landi Degl'Innocenti, E., \& Landolfi, M. 2004, Polarization in Spectral Lines (Dordrecht: Kluwer) (LL04)

Pierce, K. 2000, in Allen's Astrophysical Quantities, ed. A. N. Cox 4th edn. (New York: Springer), 355

Ralchenko, Yu., Kramida, A. E., Reader, J., \& NIST ASD Team 2008, NIST Atomic Spectra Database (version 3.1.4) (Gaithersburg, MD.: NIST) [Online] Stenflo, J. O. 1997, A\&A, 324, 344

Stenflo, J. O., \& Keller, C. U. 1996, Nature, 382, 588

Stenflo, J. O., \& Keller, C. U. 1997, A\&A, 321, 927

Trujillo Bueno, J. 2001, Atomic Polarization and the Hanle effect in Advanced Solar Polarimetry: Theory, Observations and Instrumentation, ed. M. Sigwarth, ASP Conf. Ser., 236, 161

Trujillo Bueno, J. 2003, New Diagnostic Windows on the Weak Magnetism of the Solar Atmosphere in Solar Polarization, Proceeding of the Conference held 30 September - 4 October 2002 at Tenerife, Canary Islands, Spain, ed. J. Trujillo Bueno, \& J. Sanchez Almeida, ASP Conf. Ser., 307, 407

Trujillo Bueno, J. 2009, The Magnetic Sensitivity of the Second Solar Spectrum, in Solar Polarization 5, ed. S. Berdyugina, K. N. Nagendra, \& R. Ramelli, ASP Conf. Ser., 405, 65

Trujillo Bueno, J., Casini, R., Landolfi, M., \& Landi Degl'Innocenti, E. 2002, ApJ, 566, L53

Villemoes, P., van Leeuwen, R., Arnesen, A., et al. 1992, Phys. Rev. A, 45, 6241 